\title{
Acides gras oméga-3 alimentaires et neurophychiatrie
}

Oléagineux, Corps Gras, Lipides. Volume 11, Numéro 4, 362-70, JUILLET-OCTOBRE 2004, Fondamental

Auteur(s) : Jean-Marie Bourre

Hôpital Fernand Widal, 200, rue du Faubourg Saint-Denis, 75475 Paris cedex 10

Summary : Omega-3 fatty acids participated in the first coherent experimental demonstration of the effect of dietary substances (nutrients) on the structure and function of the brain. Experiments were first of all carried out on ex-vivo cultured brain cells, then on in vivo brain cells, finally on physiochemical, biochemical, physiological, neurosensory, and behavioural parameters. These findings indicated that the nature of polyunsaturated fatty acids (in particular omega-3) present in formula milks for infants (both premature and term) determines the visual, cerebral, and intellectual abilities, as described in a recent review in OCL [1]. In view of the high omega-3 polyunsaturated fatty acid content of the brain, it is evident that these fats are involved in brain biochemistry, physiology and functionning $\backslash$; and thus in some neuropsychiatric diseases and in the cognitive decline of ageing. Though omega-3 fatty acids appear effective in the prevention of stress, their role as regulator of mood and libido is a matter for discussion pending experimental proof in animal and human models. Dietary omega-3 fatty acids could play a role in the prevention of some disorders including depression, as well as in dementia, particularly Alzheimer's disease. Their direct role in major depression and bipolar disorder (manic-depressive disease) and schizophrenia is not yet established. Their deficiency can prevent the renewal of membranes, and thus accelerate cerebral ageing〉; nonetheless, the respective roles of the vascular component on one hand (where the omega-3's are active) and the cerebral parenchyma itself on the other, have not yet been clearly resolved. The role of omega-3 in certain diseases such as dyslexia and autism is suggested. The role of omega- 3 in certain diseases such as dyslexia, autism, and schizophrenia seems to suggest a problem of diet. Indeed, the insufficient dietary supply of omega-3 fatty acids in today's French diet raises the problem of how to correct dietary habits so that the consumer will select foods that are genuinely rich in omega-3/the omega-3 family $\backslash$ mainly rapeseed and walnut oils on one hand and fatty fish on the other.

Keywords : fatty acids, omega-3, brain, psychiatry, depression, dementia, stress, mood

\section{ARTICLE}

\section{Introduction}

Les acides gras oméga-3 constituent une famille dont le premier élément est l'acide alphalinolénique indispensable (ALA, 18:3 oméga-3, principalement trouvé dans le règne végétal); les autres éléments, élaborés à partir de l'ALA, sont constitués de chaînes carbonées plus longues et plus insaturées, principalement présentes dans le monde animal, les principaux étant l'EPA (acide 
eicosapentaénoïque, dit timnodonique, 20:5 oméga-3) et le DHA (acide docosahexaénoïque, dit cervonique, 22:6 oméga-3). Ils sont l'objet d'un intérêt considérable, dépassant celui du corps médical et celui de la diététique, au point d'être utilisés à des fins de publicité. De multiples gélules sont mises sur le marché de la parapharmacie et du complément alimentaire ; avec, très récemment, des visées sur le fonctionnement cérébral de l'adulte.

Sur le plan de la santé, les oméga-3 ont été mis en exergue par la prévention et le traitement des maladies cardiovasculaires ; I'huile de chair de poisson (riche en EPA et en DHA) est un médicament dont l'indication principale est la réduction de l'hypertriglycéridémie, suite aux observations de réduction du risque cardiovasculaire chez les esquimaux. Des centaines de travaux ont été publiés dans le domaine cardiovasculaire. Globalement, les acides gras oméga-3 interviennent schématiquement dans le cadre de la prévention des maladies vasculaires, au titre d'une part de I'ALA qui lutte, par exemple, contre l'agrégation plaquettaire et l'inflammation (soit directement, soit par sa transformation en EPA), et, d'autre part des longues chaînes carbonées (EPA et DHA) qui modulent, entre autres, les triglycérides sériques; tous agissent sur la physiologie des cellules endothéliales et musculaires des parois vasculaires, sur la pression sanguine, évitent l'arythmie cardiaque, l'inflammation, etc.

D'autre part, les acides gras oméga-3 ont été valorisés à travers leur caractère indispensable dans la construction, le maintien et le fonctionnement du cerveau. Presque tous les lipides de cet organe, en particulier les phospholipides, participent aux structures des membranes biologiques, en aucun cas ils ne sont utilisés à des fins énergétiques. Ces phospholipides sont formés d'acides gras ; environ un tiers des acides gras cérébraux sont polyinsaturés. Parmi ceux-ci, approximativement un tiers (avec des variations selon les régions cérébrales) sont issus de la famille oméga-3. Les acides gras oméga-3 sont donc à prendre sérieusement en compte dans le cadre du cerveau et de ses fonctions supérieures [1], ce qui a été revu récemment dans OCL [2]. En fait, parmi les acides gras oméga-3, I'ALA a constitué la première démonstration expérimentale, cohérente et simultanée entre diverses disciplines scientifiques, de l'effet d'une substance alimentaire à destinée structurale sur la structure et la fonction du cerveau. Dans un premier temps, il a été montré que la différentiation et la fonctionnalité de cultures cellulaires de cerveau dissocié nécessitent non seulement l'ALA, mais aussi les très longues chaînes carbonées oméga-3 et oméga-6 [3]. II a ensuite été démontré que la carence alimentaire en ALA altérait le cours du développement cérébral, perturbait la composition des membranes des cellules du cerveau, neurones, oligodendrocytes, astrocytes et d'organites comme la myéline et les terminaisons nerveuses [4]. Cette carence se traduit par des dérèglements physicochimiques, induisant des perturbations biochimiques et physiologiques, elle génère des perturbations neurosensorielles et comportementales. Pendant les périodes de gestation et d'allaitement, il existe un effet-dose entre les quantités d'oméga-3 dans l'alimentation et l'accumulation de ces composés dans le cerveau, jusqu'à ce que l'optimum soit atteint, au-delà duquel les excès d'ALA alimentaires n'induisent plus aucun remaniement au niveau des lipides du cerveau [5]. En conséquence, la nature des acides gras polyinsaturés (en particulier oméga-3) trouvés dans les laits adaptés pour nourrissons (prématurés comme nés à terme) conditionne leurs capacités visuelles et cérébrales, y compris cognitives et intellectuelles. C'est pourquoi tous les laits adaptés mis sur le marché ces dernières années sont supplémentés en acides gras oméga-3, au minimum avec de l'ALA, voire avec du DHA en quantités équivalentes à celles du lait de femme, qui en contient naturellement. 
De multiples expérimentations ont été réalisées sur le cerveau de nombreux modèles animaux, portant sur les conséquences biochimiques, les paramètres physico-chimiques membranaires, les activités enzymatiques et celles de transporteurs, les neuromédiateurs, les données électrophysiologiques et comportementales. Les implications de ces acides parmi les autres acides dans le système nerveux de l'animal et de l'homme au cours de la vie (en particulier du développement et du vieillissement) ont été l'objet de nombreuses études. Elles ont été rappelées dans des revues récentes [6], notamment dans OCL [7], et ont même fait l'objet de livres grand public [8] dont I'un, très récent, consacré aux oméga-3 [9].

Il est logique que la psychiatrie se soit intéressée aux oméga-3 pour plusieurs raisons préliminaires. La richesse du cerveau en oméga-3 justifie la première. La seconde est la conséquence directe des études réalisées chez les modèles expérimentaux qui ont montré que la carence alimentaire en ALA induit des déficits comportementaux et cognitifs, en particulier au niveau de l'apprentissage $[5,10$ 13] de la mémorisation et de l'habituation [14,15], de réactions à la morphine [16] ; elle induit des anomalies du métabolisme de certains neuromédiateurs [17, 18], comme revu dans OCL [19]. Nombre de déficits peuvent être corrigés par une alimentation adaptée [20-24]. Le DHA a certainement joué un rôle important dans l'évolution du cerveau humain [25].

Toutefois, les rôles respectifs de la composante vasculaire et du parenchyme cérébral ne sont pas encore clairement définis. Ainsi, une relation est faite entre la démence et le risque cardiovasculaire, avec dans les deux cas un déficit en acides gras oméga-3, auquel s'ajoute une augmentation des concentrations en homocystéine [26]. L'hyperhomocystéinémie constitue d'ailleurs un risque psychiatrique au cours du vieillissement [27].

\section{Santé mentale, humeur, stress}

Bien que les suspicions soient nombreuses, les études strictement dédiées à l'humeur restent rares sur la relation entre acides gras (notamment oméga-3) et humeur. Ainsi, sur une cohorte néozélandaise de 4644 sujets âgés de plus de 15 ans, la perception personnelle d'un meilleur état de santé mentale et physique est en proportion de la consommation de poisson, donc d'acides gras oméga-3, qui sont de ce fait considérés comme des stabilisateurs de I'humeur [28]. En revanche, en Angleterre, une étude montre que la consommation de poissons n'améliore pas l'humeur des personnes non dépressives [29]. Pour un auteur australien, manger régulièrement des repas normaux (incluant des oméga-3) et prendre un petit-déjeuner consistant améliore l'humeur et les performances cognitives [30]. Ces acides gras participent donc à une indispensable hygiène générale de vie, plutôt qu'ils n'agissent du fait de leurs particularités biochimiques. Les rares essais de traitement de la fatigue chronique sont contradictoires [31]. Il est donc prématuré d'affirmer que les acides gras oméga-3 modulent l'humeur.

Un rapport alimentaire (en fait administré sous forme de capsules formulées à partir d'huiles végétales) optimal oméga-3/oméga-6 (égal à un quart, pour les acides alpha-linolénique et linoléique) a été défini pour lutter contre le stress. II protégerait en particulier contre les altérations de l'hippocampe lors des excès de cortisol et de corticostéroïdes [32]. Chez l'adulte jeune, l'agressivité est en proportion inverse de la consommation de poisson avec un $\mathrm{OR}=0,82$ [33]. Par ailleurs, le DHA (1,5 à 1,8 g quotidiens de DHA ; trouvé dans une huile de poisson enrichie en DHA) a une activité anti-stress, et diminue les tendances agressives chez les jeunes adultes en modulant peut-être le stress [34]. À des doses voisines administrées, pendant 2 mois à des personnes âgées de 
50 à 60 ans, l'agressivité est diminuée de $30 \%$, mais des doses de $150 \mathrm{mg}$ quotidiennes sont insuffisantes [35], c'est-à-dire que les ANC français sont insuffisants pour produire un effet. L'EPA est actif dans le traitement de femmes souffrant de troubles de la personnalité [36]. Il est intéressant de noter que les acides gras oméga-3 diminueraient la perception de la douleur, en impliquant directement les processus neuronaux et gliaux qui génèrent la douleur inflammatoire [37].

\section{Dépression}

L'accroissement de la prévalence de la dépression, depuis une cinquantaine d'années, peut être mis en parallèle avec des modifications profondes des comportements alimentaires, caractérisées en particulier par une diminution des aliments qui contiennent des acides gras oméga-3 [38]. En Colombie britannique, l'incidence de la dépression s'est accrue avec la perte des habitudes alimentaires traditionnelles caractérisées par leur richesse en poisson ; elle a ensuite diminué avec la réintroduction de ces aliments [39]. II existe une corrélation entre la diminution de la consommation d'acides gras oméga-3 (des poissons) et le risque de dépression, d'autant que l'incidence de la maladie varie de 1 à 50 selon les pays, en parallèle avec la consommation de poisson $[40,41]$; ce qui n'est pas confirmé par une autre étude [42]. En Crète, une relation inverse entre la teneur en DHA dans le tissu adipeux et le risque de dépression a été démontré [43]. Une synthèse des résultats publiés, obtenus par 41 études dans 23 pays, montre qu'une faible consommation de poisson induit une teneur basse en DHA dans le lait maternel (ce qui est défavorable pour le nourrisson) et accroît le risque de dépression post-partum chez la mère, mais il n'y a pas de relation ni avec l'EPA ni avec I'acide arachidonique [44].

En termes de témoin biologique, une augmentation du rapport sanguin acide arachidonique/EPA est en relation avec un accroissement du risque de dépression [45]. Des anomalies similaires sont retrouvées pour les esters du cholestérol et les phospholipides plasmatiques [46], comme dans les dépressions majeures [47]. Plus précisément, la sévérité de la dépression est proportionnelle à la diminution des acides gras oméga-3 dans les membranes érythrocytaires, indépendamment de l'apport calorique [48], ce qui pourrait être en relation avec des dommages oxydatifs [49]. La diminution du DHA sérique en proportion de la dépression post-partum, associée à une normalisation plus lente de la teneur en DHA du sang, fait conclure à l'intérêt de la supplémentation en EPA et DHA pendant la grossesse et la période qui suit l'accouchement [50,51]. La relation entre les modifications des acides gras et le risque de dépression du vieillissement reste à faire, d'autant qu'il a été montré une relation entre la composition plasmatique en acides gras et le risque de dépression au cours du vieillissement [52]. Une réduction de la consommation d'acides gras oméga-3 induirait un risque de dépression et de suicide, peut-être en augmentant l'activité sérotoninergique centrale et en diminuant les comportements impulsifs et agressifs [53].

En pratique thérapeutique, deux études ont montré que l'EPA sous forme d'ester éthylique, à la dose de $2 \mathrm{~g} /$ jour, serait efficace dans le traitement de dépressions incomplètement maîtrisées par traitements psychiatriques classiques [54,55]. Ajouté aux traitements conventionnels, mais sur un seul malade, I'EPA non seulement améliore les signes cliniques (tendances suicidaires, phobies sociales), mais engendre des modifications morphologiques (diminution du volume des ventricules latéraux) [56]. Le DHA lui-même est efficace pour les dépressions mineures [57]. Une seule étude, portant sur une seule malade, a montré l'efficacité des oméga-3 dans le traitement de la dépression de la grossesse et du post-partum [58], alors qu'une autre étude montre des résultats négatifs, avec 
une huile de poisson à raison de 2,69 g par jour (EPA/DHA $=1,4)$, commencé à la $34-36^{\mathrm{e}}$ semaine de grossesse, et jusqu'à 12 semaines après l'accouchement [59]. Une autre montre que la supplémentation en DHA à raison de $200 \mathrm{mg}$ par jour pendant 4 mois après l'accouchement prévient le déclin plasmatique de cet acide de leurs hématies, mais ne modifie pas leur auto-évaluation de dépression [60] ; mais ce résultat s'expliquerait par le fait qu'il s'agit vraisemblablement de mesures de modulation de l'humeur plutôt que de dépression réelle, et d'autre part ces doses sont probablement trop faibles. Le traitement par les acides gras oméga-3 (à raison de 9,6 g quotidiens pendant 8 semaines) a montré des résultats positifs dans les cas de dépression majeure [61], mais le DHA seul (à raison de $2 \mathrm{~g} /$ jour, pendant 6 semaines) ne semble pas efficace [62]. En tout état de cause, l'EPA semble augmenter l'action des antidépresseurs [63].

Chez les maniaco-dépressifs, une étude dans 14 pays montre qu'il existe une corrélation entre la prévalence de la maladie et la moindre consommation de poisson, le seuil de vulnérabilité se situant à $65 \mathrm{~g} / \mathrm{jour}$ [64] ; le traitement avec des acides gras oméga-3 pourrait être utile dans des conditions bien précises [65].

Quoi qu'il en soit, les traitements classiques par les anti-dépresseurs ne s'accompagnent pas, semblet-il, de la normalisation des acides gras oméga-3 [66]. Les mécanismes d'action sont donc probablement différents.

Les acides gras oméga-3 ne concernent très probablement pas (au moins de manière directe) les affections somatiques dépressogènes neurologiques (maladie de Parkinson, tumeur, épilepsie temporale, traumatismes craniaux-cérébraux) ou endocriniennes (hypo- ou hyperthyroïdie, etc.) ; sans négliger la déficience en vitamine B12 (et dans une moindre mesure en folates, vitamine B9), dont les symptômes psychiatriques précèdent les signes hématologiques. En revanche, il n'est pas illusoire que les acides gras oméga-3 puissent être intéressants pour agir sur la dépression hivernale saisonnière, car ils modulent chez l'animal certaines activités hypophysaires, en particulier la sécrétion de mélatonine [67].

\section{Démences}

Quelques études épidémiologiques ont montré l'intérêt des acides gras oméga-3 dans le cadre de la prévention des démences. Ainsi, dans l'étude dite de Rotterdam, le risque de démence avec composante vasculaire est positivement corrélé avec la consommation de graisses saturées, inversement avec celle de poisson riche en acides gras oméga-3 [68], observation non confirmée par une autre étude [69]. Plus spécifiquement, une forte consommation d'acides gras insaturés et de graisses non-hydrogénées protégerait contre la maladie d'Alzheimer, le contraire étant observé avec les graisses saturées et les acides gras trans [70]. La consommation de poisson sauvage exerce un effet protecteur contre les démences, y compris d'Alzheimer. En France, la consommation de viande, du fait de ses acides gras saturés, serait peu en relation avec une augmentation du risque de démence, la consommation de poisson sauvage exerçant manifestement un effet inverse, c'est-àdire protecteur. Cette conclusion a été formulée à la suite de l'examen des participants à la cohorte dite « PAQUID » portant sur 1416 personnes âgées de plus 67 ans habitant dans le sud-ouest, suivies pendant 7 ans; elle montre l'apparition de 170 démences, dont 135 de type d'Alzheimer ; ceux qui mangent du poisson au moins une fois par semaine présentent un risque diminué de $34 \%$ pour toutes les démences quelle qu'en soit la forme, et de $31 \%$ pour la démence d'Alzheimer ; l'effet persiste si le niveau socio-culturel est pris en compte, car ce dernier est en relation avec, d'une part, 
la diminution du risque de maladie d'Alzheimer et, d'autre part, avec la consommation de poisson [71]. Aux États-Unis, une étude a montré que la prévalence de la maladie d'Alzheimer est diminuée de $60 \%$ chez les personnes qui consomment environ 60 mg de DHA par jour (trouvés dans au moins une part de poisson par semaine) par comparaison avec ceux qui en consomment très peu [72]. Au Japon, il en est globalement de même [73].

Pour ce qui concerne les témoins biologiques, des teneurs plasmatiques basses en acides gras oméga-3 (y compris en DHA) constituent des facteurs de risque pour le déficit cognitif et divers types de démences, y compris celle d'Alzheimer [74]. En revanche, dans une seule étude, les acides gras oméga-3 sériques ne sont pas altérés [75].

Il existe des facteurs nutritionnels communs entre la démence vasculaire et la maladie d'Alzheimer : un excès d'acides gras oméga- 6 et un déficit en acides gras oméga-3, qui induisent des altérations de la micro-vascularisation, une inflammation chronique, une agrégation plaquettaire et des dysfonctionnements endothéliaux [76]. Cette proposition expliquerait, au moins partiellement, que les perturbations cognitives chez l'homme très âgé sont positivement corrélées avec la consommation d'acide linoléique, et négativement avec celle de poisson [68]. En fait, il n'a pas encore été publié de traitements préventifs de la démence avec des acides gras oméga-3. Le risque cardiovasculaire augmente celui de démence, en particulier vasculaire [77]. Dans tous les cas, des mécanismes inflammatoires pourraient être impliqués [78]

Un mélange d'ALA et d'acide linoléique dans un rapport quantitatif de un quart (administré sous forme de capsules dans l'étude en question) améliore la qualité de la vie de malades alzhémériens, mesurée par des tests divers (orientation spatiale, coopération, humeur, appétit, mémoire à court et à long terme, hallucinations, sommeil) [79].

L'implication des acides gras oméga-3 dans l'alcoolisme et la démence alcoolique n'a pas été étudiée, bien qu'il ait été démontré que la présence nutritionnelle d'ALA module les effets de l'alcool, par exemple au niveau des terminaisons nerveuses de l’animal expérimental [80].

\section{Schizophrénie}

Des altérations dans le métabolisme des phospholipides ont été hypothétiquement impliquées dans la genèse de la schizophrénie, comme prix à payer dans I'humanisation, compte tenu de la quantité de phospholipides riches en acides gras oméga-3 dans le cerveau [81]. Les schizophrènes qui absorbent le plus de poisson présentent des signes cliniques moins sévères [82].

Les concentrations en DHA et EPA sont diminuées dans les membranes des érythrocytes des malades schizophréniques, sans qu'il y ait de relation entre le degré d'altération du profil en acides gras et la gravité des signes cliniques; les différences ne sont pas dues au sexe, à l'état hormonal ou à l'usage du cannabis [83]. De tels résultats n'ont pas été retrouvés dans une autre étude, le tabagisme y semblant alors un facteur discriminant [84] ; en fait, il pourrait exister des sous-groupes de malades chez lesquels les oméga-3 seraient plus particulièrement concernés, ce qui expliquerait que le même groupe de cliniciens ait trouvé des résultats disparates, avec pour la teneur de DHA érythrocytaire soit une augmentation, soit une diminution, soit une absence d'effet, selon les publications. Des anomalies dans le renouvellement des phospholipides ont été évoquées dans la schizophrénie [85]. 
Le traitement avec une huile de poisson ( $10 \mathrm{~g} / \mathrm{jour}$ pendant 6 semaines) améliore les symptômes [86] ; tout comme le fait pendant 4 mois, mais de manière relativement modeste, la combinaison biquotidienne d'EPA (120 mg), de DHA (150 mg) et de vitamines C (500 mg) et E (400 UI) [87]. L'utilisation spécifique d'EPA chez un seul malade exempt de tout traitement a amélioré les symptômes, régularisé le renouvellement des phospholipides cérébraux (déterminé par RMN au 31phosphore) et même fait rétrocéder l'atrophie cérébrale en 6 mois de traitement [88] ; ce résultat demande évidemment à être confirmé. Le traitement durant 3 mois chez des patients traités et stabilisés est intéressant [89], voire même en supplément des traitements anti-psychotiques ayant duré au moins 6 mois, mais laissant des symptômes résiduels [90]. Toutefois, pour un auteur, l'EPA ne donnerait aucun résultat à la dose de $3 \mathrm{~g} / \mathrm{jour}$ [91]. Cet échec pourrait s'expliquer par des effets de doses, ou bien par la sélection de groupes de patients particuliers, non-répondeurs [92]. De multiples théories pourraient expliquer la participation des acides gras oméga-3 dans la schizophrénie [93], d'autres font intervenir la modulation des neurotransmissions, en particulier dopaminergiques, mais sans preuve de leurs implications pour le moment [89].

\section{Handicaps, maladies neurologiques, vieillissement cognitif}

Les enfants handicapés neurologiques absorbent insuffisamment d'acides gras oméga-3, comme le montre dans leur sérum la présence des marqueurs de carence que sont le 20:3 oméga-9 et le 22:5 oméga-6, ce qui n'est pas favorable au bon renouvellement de leurs structures cérébrales déjà atteintes [94]. Les altérations observées dans certaines maladies, comme la céroïde lipofuscinose neuronale infantile associée à une démence, sont secondaires [95]. Les effets favorables du DHA dans le cadre du traitement de la maladie péroxysomale de Zellweger [96] ne constituent qu'une compensation d'un métabolisme déficient.

Les acides gras polyinsaturés, dont les acides gras oméga-3, seraient impliqués dans les maladies du développement cérébral, les déficits d'attention et les hyperactivités, les dyslexies et même l'autisme [97]. Chez l'enfant dyslexique, la sévérité des signes est en proportion du déficit en acides gras polyinsaturés, mais chez les garçons seulement [98]. Un groupe de 135 adultes (74 hommes et 61 femmes), la dyslexie est accompagnée de signes de déficience en acides gras polyinsaturés [99]. Les acides gras sembles occuper une place importante dans les déficits en attention associés à une hyperactivité [100] ; une augmentation de $50 \%$ de la dégradation oxydative des acides gras oméga-3 est observée, mais pas de celle des protéines [101]. Une étude pilote portant sur 50 enfants montre que la supplémentation en acides gras indispensables (prise quotidienne de $480 \mathrm{mg}$ de DHA, $80 \mathrm{mg}$ d'EPA, de $40 \mathrm{mg}$ d'acide arachidonique, de $96 \mathrm{mg}$ d'acide gamma-linolenique et de $24 \mathrm{mg}$ d'acétate de tocopherol) améliore la symptomatologie [102]. Dans une autre étude, la supplémentation améliore les paramètres sanguins, mais pas les symptômes cliniques des enfants hyperactifs souffrant de déficit d'attention [103].

Une étude française a montré une diminution de $23 \%$ du DHA dans les phospholipides plasmatiques d'enfants autistes, le total des acides gras oméga-3 étant diminué de $20 \%$, alors que les acides gras oméga-6 ne sont pas affectés [104]; une diminution de $70 \%$ est aussi trouvée dans les phospholipides des globules rouges [105]. Le traitement par l'EPA (à raison de $540 \mathrm{mg} / \mathrm{jour}$ pendant 4 semaines) trouvé dans une huile de poisson, a traité un jeune homme autiste âgé de 11 ans [106]. D'autre part, la transduction des signaux en relation avec les phospholipides est normale chez ces malades [107]. 
Dans le domaine des toxicomanies, l'implication des oméga-3 (et d'ailleurs aussi des oméga-6) n'est pas à exclure. En effet, la rechute à la cocaïne est d'autant plus rapide que le sujet est déficitaire en acides gras polyinsaturés $[108,109]$. II est possible de proposer que l'altération des membranes biologiques de certains neurones cérébraux, pour des causes alimentaires, induise une plus grande fragilité de certains sujets, par ailleurs déjà éventuellement prédisposés à la toxicomanie, pour d'autres raisons. En tout état de cause, l'état nutritionnel des toxicomanes est fréquemment très mauvais, Dans quelle mesure peut-il aggraver leurs pathologies, nuire à l'efficacité du sevrage ou augmenter les rechutes ? Voilà une question de grande importance, d'autant que l'expérimentation animale montre que la carence en acide alpha-linolénique altère les réponses à la morphine [110].

L'intérêt des acides gras insaturés dans le cadre de la prévention et de l'accompagnement du traitement de la sclérose en plaques est évoqué depuis longtemps; ne serait-ce que pour assurer les besoins en acides gras polyinsaturés lors de la reconstruction de la gaine de myéline. La supplémentation alimentaire permettrait une diminution de la sévérité et de la fréquence des rechutes, au moins sur une période de 2 ans [111], d'autant qu'une diminution de la concentration plasmatique et érythrocytaire de l'acide linoléique a été notée [112]. Dans le cadre de la diminution du nombre et de l'intensité de poussées de la maladie, l'intérêt spécifique des acides gras oméga-3 à raison de $0,9 \mathrm{~g} / \mathrm{jour}$ sous forme d'huile de poisson pendant 2 ans [113] pourrait résider dans la modulation des cytokines [114].

Les modifications observées au cours du vieillissement tant chez les modèles animaux que chez l'homme sont complexes, selon les régions, les structures, les cellules, les organites et les lipides considérés ; les acides gras oméga-3 sont concernés, directement ou indirectement [6]. II est à noter que des docosanoïdes ont été mis en évidence dans le système nerveux $[115,116]$. Un mécanisme d'action particulier des acides gras oméga-3, et notamment du DHA, concerne les régulations des ATPases : d'une part, parce qu'environ $60 \%$ de l'énergie cérébrale est en relation avec ces enzymes [117] et, d'autre part, parce que les isoformes de ces enzymes sont modulées par les acides gras oméga-3, soit l'ALA [118], soit le DHA et I'EPA des huiles de poisson [119]. Par ailleurs, le métabolisme péroxysomal est impliqué, en particulier au niveau des acides gras polyinsaturés [120]. En France, une étude très récente a montré que le déficit cognitif du vieillissement dans la population est en relation avec la diminution du rapport oméga-3/oméga-6 des érythrocytes [121] ; un excès nutritionnel en acide linoléique est en relation avec le déclin des performances cognitives, alors que c'est l'inverse avec les huiles de poisson [68,122].

Une augmentation du stress oxydant lors du vieillissement, dû à une diminution des protections contre les radicaux libres, peut induire une diminution des acides gras oméga-3 dans les structures nerveuses, et de ce fait une alimentation enrichie en EPA pourrait présenter des propriétés antioxydantes qui permettraient de lutter contre les effets du vieillissement [123]. En fait, la diminution des teneurs en acides gras polyinsaturés dans les structures cérébrales peut résulter soit d'une diminution des transferts à travers la barrière hémato-encéphalique, soit d'une réduction de leur incorporation dans les membranes, soit d'un amoindrissement des activités des delta-6 ou delta- 9 désaturases; mécanismes auxquels s'ajoute le stress oxydatif qui augmente la production des radicaux libres et par conséquent perturbe les fluidités membranaires [124]. La phosphatidylcholine améliore la mémoire, l'apprentissage, la concentration, le mémoire des mots et l'humeur chez les personnes âgées en déclin cognitif [125]. Associée à la vitamine B12, la phosphatidylcholine améliore les performances d'apprentissage, au moins chez la souris vieillissante [126]. Mais il reste certain 
qu'un approvisionnement adéquat en acides gras oméga-3 assure un bon renouvellement des membranes, et protège donc, dans une certaine mesure, contre le vieillissement cérébral. La supplémentation, avec uniquement de fortes quantités d'acides gras oméga-3, induit des modifications comportementales qui ne sont pas les mêmes selon les âges de la vie, augmentant l'apprentissage chez les animaux jeunes; mais le diminuant, ainsi que l'activité motrice, chez les âgés [127]. Cette observation est à prendre en compte dans les indications de supplémentations.

Pour ce qui touche au vieillissement, comme en ce qui concerne de multiples maladies neurologiques ou psychiatriques, dans le cadre spécifique de la physiologie membranaire, de nombreuses études recherchent (sur les modèles animaux et chez l'homme) des anomalies dans le métabolisme des lipides (notamment des phospholipides), soit dans le cerveau, soit dans d'autres tissus, y compris la peau, ou celui des régulations au niveau des messagers lipidiques. N'étant pas en relation directe avec l'alimentation, il ne sont pas référencés dans cette revue, mais le sont ailleurs $[1,6]$.

\section{Conclusions}

Très schématiquement, il convient de distinguer deux modalités d'action des acides gras, notamment oméga-3 : l'une sur le long terme, l'autre sur le court terme. Pour le long terme, ils agissent sur les compositions des membranes, et donc sur leurs fonctions; cette hypothèse est étayée par de nombreuses études, en particulier celles portant sur le développement cérébral et celles montrant la prévention de la démence, y compris celle d'Alzheimer, par les acides gras oméga-3 alimentaires. Pour le court terme, il est possible de proposer que les acides gras interviennent par l'intermédiaire du métabolisme des phospholipides et donc, entre autres, par la modulation des transductions des signaux; cet hypothèse est étayée par l'action suggérée de I'EPA (dépression, schizophrénie, autisme). Les deux modalités d'action peuvent survenir simultanément, comme l'inflammation lors de la maladie d'Alzheimer. II reste cependant prématuré d'affirmer que les acides gras oméga-3 préviennent la dépression en traitant l'inflammation. En effet, il est évident que des signes biologiques d'inflammation sont présents lors de la dépression; il est par ailleurs exact que les acides gras oméga-3 préviennent et traitent dans une certaine mesure ces signes. Mais il ne peut exister que des relations de concomitance, et non pas de causalité.

Ces considérations amènent à distinguer fondamentalement deux conceptions d'implication des acides gras au niveau de la physiologie du cerveau. La première concerne les effets des acides gras alimentaires, notamment oméga-3, sur l'élaboration et le maintien des structures cérébrales, par conséquent sur leur fonctionnement. La deuxième met en cause des mécanismes physiologiques dans lesquels ces acides gras sont partie prenante.

Très globalement, à ce jour, pour ce qui concerne l'intervention des acides gras oméga-3 en psychiatrie, la majorité des résultats obtenus portent sur la prévention par la consommation de poisson gras (notamment de dépression, de démence et de maladie bipolaire). La principale caractéristique de ces poissons gras est d'être riches en oméga-3, mais d'autres composants peuvent également être responsables d'effets favorables (iode dont l'impact sur le cerveau est considérable, sélénium, entre autres). Par des essais cliniques rigoureux, il reste encore à apporter la preuve incontestable de l'efficacité de la prescription de gélules contenant ces oméga-3 sous forme d'huile de chair de poisson. II en est de même de la prescription d'oméga-3 purifiés, généralement sous 
forme d'esters éthyliques, qui ne repose encore que sur beaucoup trop peu d'expérimentations ou d'essais cliniques pour être concluante, donc crédible. Attention donc aux gélules et capsules. En effet, les oméga-3 font partie de constructions moléculaires, naturelles et biodisponibles pour le corps humain, les triglycérides et les phospholipides. Par contre, l'enrichissement en tel ou tel acide gras oméga-3 nécessite la destruction de cette construction, puis la liaison des oméga-3 le plus souvent, à de l'alcool, formant donc un ester éthylique : il ne s'agit plus alors d'un produit naturel, mais d'une substance chimique, dont il n'est pas prouvé qu'elle soit un médicament, ni même qu'elle soit active. La sécurité est donc de rechercher les indispensables oméga-3 dans les aliments qui les contiennent naturellement.

La question est de connaître les aliments réellement riches en oméga-3. Compte tenu notamment des recommandations de l'AFSSA [128] et celles des ANC (apports nutritionnels conseillés pour la population française [129]), il convient de sélectionner en priorité, parmi les aliments destinés à la consommation humaine, ceux qui apportent plusieurs décigrammes quotidiens d'ALA. En effet, l'absorption moyenne est inférieure à $1 \mathrm{~g}$ quotidien, alors que les ANC journaliers sont, respectivement, de 2 et de 1,6 $\mathrm{g}$ pour les hommes et les femmes adultes. Schématiquement, en alimentation humaine usuelle en France, il faudrait privilégier les huiles de colza (au premier chef), de noix (mais elle est plus onéreuse que celle de colza), de soja (mais elle contient de l'acide linoléique, oméga-6, en grande quantité). Les noix sont intéressantes. En ce qui concerne les très longues chaînes carbonées oméga-3, il s'agit des poissons gras sauvages et d'élevage, à la condition express que leur nourriture ait été satisfaisante, préoccupation qui a fait l'objet d'une revue récente dans OCL [7] ; ainsi que les œufs " oméga-3 » ou récemment « bénéfic " (et non, généralement, les œufs label ou bio). Les ANC quotidiens pour le DHA sont respectivement de 120 et de $100 \mathrm{mg}$ pour les hommes et les femmes adultes. Le facteur de bio-équivalence de 10 qui permet de convertir les apports en DHA et EPA en ALA est généralement retenu, mais il est en fait variable selon les sexes, les âges de la vie et les situations physiologiques [130-133].

Toutefois, accroître la quantité d'acides gras oméga-3 dans les aliments n'est pas suffisant, bien que fondamental. II faut tenir compte de la présence d'acides gras oméga-6, afin que le rapport oméga6/oméga-3 se rapproche des recommandations, c'est-à-dire de 5 . Or, ce rapport est beaucoup trop élevé dans l'alimentation de la population française. II ne sera efficacement abaissé que par l'augmentation quantitative des acides gras oméga-3; mais, certainement pas, avec une diminution des oméga-6, dont les apports ne sont que légèrement supérieurs aux recommandations. Les aliments dont le rapport est inférieur à 5 seront qualifiés d'équilibrants (car corrigeant un rapport trop grand dans l'alimentation usuelle des français; I'huile de colza constituant le prototype), ceux dont le rapport sera proche de 5 seront qualifiés d'équilibrés.

\section{Références}

1. BOURRE JM. Effets des nutriments (des aliments) sur les structures et les fonctions du cerveau : le point sur la diététique du cerveau. Rev Neurol $2004 ; 160$ : 767-92.

2 BOURRE JM. Relations entre acides gras oméga-3, oméga-9, structures et fonctions du cerveau. Le point sur les dernières données. Le coût financier alimentaire des oméga-3. OCL $2003 ; 3: 165-74$. 
3 BOURRE JM, FAIVRE A, DUMONT O, et al. Effect of polyunsaturated fatty acids on fetal mouse brain cells in culture in a chemically defined medium. J Neurochem $1983 ; 41: 1234-42$.

4 BOURRE JM, PASCAL G, DURAND G, MASSON M, DUMONT O, PICIOTTI M. Alterations in the fatty acid composition of rat brain cells (neurons, astrocytes and oligodendrocytes) and of subcellular fractions (myelin and synaptosomes) induced by a diet devoided of ( $n-3)$ fatty acids. J Neurochem $1984 ; 43: 342-8$.

5 BOURRE JM, FRANÇOIS M, YOUYOU A, et al. The effects of dietary alpha-linolenic acid on the composition of nerve membranes, enzymatic activity, amplitude of electrophysiological parameters, resistance to poisons and performance of learning task in rat. J Nutr $1989 ; 119: 1880-92$.

6 BOURRE JM. Roles of unsaturated fatty acids (especially omega- 3 fatty acids) in the brain at various ages and during ageing. J Nutr Health and Aging $2004 ; 3$ : 163-74.

7 BOURRE JM. Alimentation animale et valeur nutritionnelle induite sur les produits dérivés consommés par l'homme. Les lipides sont-ils principalement concernés ? OCL 2003 ; 5 : 405-24.

8 BOURRE JM. La diététique du cerveau. Paris France : Editions Odile Jacob, 1990 ; De la inteligencia y el placer, la dietetica del cerebro, Biblioteca Mondatori 1991. Intelligenz und Ernährung, Econ Verlag, Germany (1992). La dietetica del cervello, Sperling Kupfer, Italy (1992). Comida inteligente, a dietética do cérebro, Gradiva (1993). Brainfood, Little Brown, USA (1993). Diététique du cerveau : la nouvelle donne (2003). Editions Odile Jacob. (Paris) France.

9 BOURRE JM. La vérité sur les oméga-3. Paris : Editions Odile Jacob, 2004.

10 YAMAMOTO N, OKANIWA Y, MORI S, NOMURA M, OKUYAMA H. Effects of a high-linoleate and a high-alpha-linolenate diet on the learning ability of aged rats. Evidence against an autoxidationrelated lipid peroxide theory of aging. J Gerontol $1991 ; 46$ : B17-B22.

11 LIM S, SUZIKI H. Changes in maze behavior of mice occur after sufficient accumulation of docosahexaenoic acid in brain. J Nutr $2001 ; 131$ : 319-24.

12 SALEM N, MORIGUCHI T, GREINER RS, et al. Alterations in brain function after loss of docosahexaenoate due to dietary restriction of n-3 fatty acids. J Mol Neurosci $2001 ; 16$ : 299-307.

13 WAINWRIGHT PE. Dietary essential fatty acids and brain function : a developmental perspective on mechanisms. Proc Nutr Soc $2002 ; 61$ : 61-9.

14 FRANCES H, COUDEREAU JP, SANDOUK P, CLEMENT M, MONIER C, BOURRE JM. Influence of a dietary alpha-linolenic acid deficiency on learning in the Morris water maze and on the effects of morphine. Eur J Pharmacol 1996 ; $298: 217-25$.

15 FRANCES H, DRAI P, SMIRNOVA M, CARRIÉ I, DEBRAY M, BOURRE JM. Nutritional ( $n-3)$ polyunsaturated fatty acids influence the behavioral responses to positive events in mice. Neurosci Lett $2000 ; 285: 223-7$.

16 FRANCES $\mathrm{H}$, MONIER C, CLEMENT M, LECORSIER A, DEBRAY M, BOURRE JM. Effect of dietary alpha-linolenic acid deficiency on habituation. Life Sci $1996 ; 58$ : 1805-16. 
17 CHALON S, VANCASSEL S, ZIMMER L, GUILLOTEAU D, DURAND G. Polyunsaturated fattry acids and central cerebral functions : focus on monoaminergic neurotransmission. Lipids 2001 ; 36 : 937-44.

18 KODAS E, PAGE G, ZIMMER L, et al. Neither the density nor function of striatal dopamine transporters were influenced by chronic $n-3$ polyunsaturated fatty acid deficiency in rodents. Neurosci Lett $2002 ; 321$ : 95-9.

19 CHALON S. Acides gras polyinsaturés et fonctions cognitives. OCL $2001 ; 4$ : 317-20.

20 CARRIE I, CLEMENT M, De JAVEL D, FRANCES H, BOURRE JM. Specific phospholipid fatty acid composition of brain regions in mice. Effects of $n-3$ polyunsaturated fatty acid deficiency and phospholipid supplementation. J Lipid Res $2000 ; 41$ : 465-72.

21 CARRIE I, CLEMENT M, De JAVEL D, FRANCES H, BOURRE JM. Phospholipid supplementation reverses behavioral and biochemical alterations induced by $n-3$ polyunsaturated fatty acid deficiency in mice. J Lipid Res $2000 ; 41: 473-80$.

22 CARRIE I, SMIRNOVA M, CLEMENT M, De JAVEL D, FRANCES H, BOURRE JM. Docosahexaenoic acid-rich phospholipid supplementation : effect on behavior, learning ability, and retinal function in control and n-3 polyunsaturated fatty acid deficient old mice. Nutr Neurosci $2002 ; 5:$ 43-52.

23 IKKEMOTO A, OHISHI M, SATO Y, et al. Reversibility of n-3 fatty acid deficiency-induced alterations of learning behavior in the rat : level of n-6 fatty acids as another critical factor. J Lipid Res $2001 ; 42$ : 1655-63.

24 KODAS E, VANCASSEL S, LEJEUNE B, GUILLOTEU D, CHALON S. Reversibility of n-3 fatty acid deficiency-induced changes in dopaminergic neurotransmission in rats : critical role of developmental stage. J Lipid Res $2002 ; 43: 1209-19$.

25 CRAWFORD M, BLOOM M, CUNNANE S, et al. Docosahexaenoic acid and cerebral evolution. Word Rev Nur Diet $2001 ; 88: 6-17$.

26 SEVERUS WE, LITTMAN AB, STOLL AL. Omega-3 fatty acids, homocysteine, and the increased risk of cardiovascular mortality in major depressive disorder. Harv Rev Psychiatry $2001 ; 9$ : 280-93.

27 REUTENS S, SACHDEV P. Homocysteine in neuropsychiatric disorders of the elderly. Int J Geriatr Psychiatry $2002 ; 17: 859-64$.

28 SILVERS KM, SCOTT KM. Fish consumption and self-reported physical and mental health status. Public Health Nutr $2002 ; 5: 427-31$.

29 NESS AR, GALLACHER JE, BENETT PD, et al. Advice to eat fish and mood : a randomised controlled trial in men with angina. Nutr Neurosci $2003 ; 6: 63-5$.

30 LOMBARD CB. What is the role of food in preventing depression and improving mood, performance and cognitive function? Med J Aust $2000 ; 173$ : S104-S105. 
31 WARREN G, McKENDRICK M, PEET M. The role of essential fatty acids in chronic fatigue syndrome. A case-controlled study of red-cell membrane essential fatty acids (EFA) and a placebo-controlled treatment study with high dose of EFA. Acta Neurol Scand 1999 ; 99 : 112-6.

32 YEHUDA S, RABINOVITZ S, CARASSO RL, Mostofsky DI. Fatty acid mixture counters stress changes in cortisol, cholesterol, and impair learning. Int J Neurosci $2000 ; 101: 73-87$.

33 IRIBARREN C, MARKOVITZ JH, JACOBS DR, SCHREINER PJ, DAVIGLUS M, HIBBELN JR. Dietary intake of n-3, n- 6 fatty acids and fish : Relationship with hostility in young adults-the CARDIA study. Eur J Clin Nutr $2004 ; 58: 24-31$.

34 HAMAZAKI T, ITOMURA M, SAWAZAKI S, NAGAO Y. Anti-stress effects of DHA. Biofactors 2000 ; $13: 41-5$.

35 HAMAZAKI T, THIENPRASERT A, KHEOVICHAIi K, SAMUHASENEETOO S, NAGASAWA T, Watanabe $S$. The effect of docosahexaenoic acid on aggression in elderly Thai subjects--a placebocontrolled double-blind study. Nutr Neurosci $2002 ; 5$ : 37-41.

36 ZANARINI MC, FRANKENBURG FR. Omega-3 Fatty acid treatment of women with borderline personality disorder : a double-blind, placebo-controlled pilot study. Am J Psychiatry $2003 ; 160$ : 167-9.

37 SHAPIRO H. Could n-3 polyunsaturated fatty acids reduce pathological pain by direct actions on the nervous system? Prostaglandins Leukot Essent Fatty Acids $2003 ; 68: 219-24$.

38 COLIN A, REGGERS J, CASTRONOVO V, ANSSEAU M. Lipids, depression and suicide. Encephale $2003 ; 29: 49-58$.

39 BATES D. Racially determined abnormal essential fatty acid and prostaglandin metabolism and food allergies linked to autoimmune, inflammatory, and psychiatric disorders among coastal British Columbia indians. Med Hyptheses $1988 ; 25$ : 103-9.

40 HIBBELN JR, SALEM N. Dietary polyunsaturated fatty acids and depression : when cholesterol does not satisfy. Am J Clin Nutr $1995 ; 62: 1-9$.

41 TANSKANEN A, HIBBELN JR, TUOMILEHTO J, et al. Fish consumption and depressive symptoms in the general population in Finland. Psychiatr Serv $2001 ; 52: 529-31$.

42 HAKKARAINEN R, PARTONEN T, HAUKKA J, VIRTAMO J, Albanes D, Lonnqvist J. Is low dietary intake of omega-3 fatty acids associated with depression? Am J Psychiatry 2004 ; 161 : 567-9.

43 MAMALAKIS G, TORNARITIS M, KAFATOS A. Depression and adipose essential polyunsaturated fatty acids. Prostaglandins Leukot Essent Fatty Acids $2002 ; 67$ : 311-8.

44 HIBBELN JR. Seafood consumption, the DHA content of mothers' milk and prevalence rates of postpartum depression : a cross-national, ecological analysis. J Affect Disord 2002 ; 69 : 15-29. 
45 ADAMS PB, LAWSON S, SANIGORSKI A, SINCLAIR AJ. Arachidonic acid to eicosapentaenoic acid ratio in blood correlates positively with clinical symptoms of depression. Lipids 1996 ; 31 : S157-S161.

46 MAES M, CHRISTOPHE A, DELANGHE J, ALTAMURA C, NEELS H, MELTZER HY. Lowered omega3 polyunsaturated fatty acids in serum phospholipids and cholesteryl esters of depressed patients. Psychiatry Res $1999 ; 85: 275-2751$.

47 MAES M, SMITH R, CHRISTOPHE A, COSYNS P, DESNYDER R, MELTZER H. Fatty acid composition in major depression : decreased omega 3 fractions in cholesteryl esters and increased $C 20: 4$ omega 6/C20 : 5 omega 3 ratio in cholesteryl esters and phospholipids. J Affect Disord $1996 ; 38: 35-46$.

48 EDWARDS R, PEET M, SHAY J, HORROBIN D. Omega-3 polyunsaturated fatty acid levels in the diet and in red blood cell membranes of depressed patients. J Affect Disord $1998 ; 48: 149-55$.

49 PEET M, MURPHY B, SHAY J, HORROBIN D. Depletion of omega-3 fatty acid levels in red blood cell membranes of depressive patients. Biol Psychiatry $1998 ; 43: 315-9$.

50 OTTO SJ, De GROOT RH, HORNSTRA G. Increased risk of postpartum depressive symptoms is associated with slower normalization after pregnancy of the functional docosahexaenoic acid status. Prostaglandins Leukot Essent Fatty Acids $2003 ; 69$ : 237-43.

51 DE VRIESE SR, CHRISTOPHE AB, MAES M. Lowered serum n-3 polyunsaturated fatty acid (PUFA) levels predict the occurrence of postpartum depression : further evidence that lowered n-PUFAs are related to major depression. Life Sci $2003 ; 73: 3181-7$.

52 TIEMEIER H, VAN TUIJL HR, HOFMAN A, KILIAN AJ, Breteler MM. Plasma fatty acid composition and depression are associated in the elderly : the Rotterdam Study. Am J ClinNutr $2003 ; 78$ : 40-6.

53 BRUNNER J, PARHOFER KG, SCHWANDT P, BRONISH T. Cholesterol, essential fatty acids, and suicide. Pharmacopsychiatry $2002 ; 35: 1-5$.

54 PEET M, HORROBIN DF. A dose-ranging study of the effects of ethyl-eicosapentaenoate in patients with ongoing depression despite apparently adequate treatment with standard drugs. Arch Gen Psychiatry $2002 ; 59$ : 913-9.

55 NEMETS B, STAHL Z, BELMAKER RH. Addition of omega-3 fatty acid to maintenance medication treatment for recurrent unipolar depressive disorder. Am J Psychiatry $2002 ; 159: 477-9$.

56 PURI BK, COUNSELL SJ, HAMILTON G, RICHARDSON AJ, HORROBIN DF. Eicosapentaenoic acid in treatment-resistant depression associated with symptom remission, structural brain changes and reduced neuronal phospholipid turnover. Int J Clin Pract $2001 ; 55$ : 560-3.

57 MISCHOULON D, FAVA M. Docosahexanoic acid and omega-3 fatty acids in depression. Psychiatr Clin North Am $2000 ; 23$ : 785-94.

58 CHIU CC, HUANG SY, SHEN WW, SU KP. Omega-3 fatty acids for depression in pregnancy. Am J Psychiatry $2003 ; 160: 385$. 
59 MARANGELL LB, MARTINEZ JM, ZBOYAN HA, CHONG H, PURYEAR L. Omega-3 fatty acids for the prevention of postpartum depression : negative data from a preliminary, open-label pilot study. Depress Anxiety $2004 ; 19: 20-3$.

60 LLORENTE AM, JENSEN CL, VOIGT RG, FRALEY JK, BERRETTA MC, HEIRD WC. Effect of maternal docosahexaenoic acid supplementation on postpartum depression and information processing. Am J Obstet Gynecol $2003 ; 188$ : 1348-53.

61 SU KP, HUANG SY, CHIU CC, SHEN WW. Omega-3 fatty acids in major depressive disorder. A preliminary double-blind, placebo-controlled trial. Eur Neuropsychopharmacol $2003 ; 13: 267-71$.

62 MARANGELL LB, MARTINEZ JM, ZBOYAN HA, KERTZ B, KIM HF, PURYEAR LJ. A double-blind, placebo-controlled study of the omega-3 fatty acid docosahexaenoic acid in the treatment of major depression. Am J Psychiatry 2003 ; 160 : 996-8.

63 MURCK H, SONG C, HORROBIN DF, UHR M. Ethyl-eicosapentaenoate and dexamethasone resistance in therapy-refractory depression. Int J Neuropsychopharmacol 2004 ; 7 : 341-9.

64 NOAGHIUL S, HIBBELN JR. Cross-national comparisons of seafood consumption and rates of bipolar disorders. Am J Psychiatry $2003 ; 160$ : 2222-7.

65 STOLL AL, SEVERUS WE, FREEMAN MP, et al. Omega 3 fatty acids in bipolar disorder : a preliminary double-blind, placebo-controlled trial. Arch Gen Psychiatry 1999 ; 56 : 407-12.

66 COPPEN A, BAILEY J. Enhancement of the antidepressant action of fluoxetine by folic acid : a randomised, placebo controlled trial. J Affect Disord 2000 ; 60 : 121-30.

67 ZAOUALI-AJINA M, GHARIB A, DURAND G, et al. Dietary docosahexaenoic acid-enriched phospholipids normalize urinary melatonin excretion in adult $(n-3)$ polyunsaturated fatty aciddeficient rats. J Nutr $1999 ; 129: 2074-80$.

68 KALMIJN S, LAUNER LJ, OTT A, WITTEMAN JC, HOFMAN A, BRETELER MM. Dietary fat intake and the risk of incident dementia in the Rotterdam Study. Ann Neurol $1997 ; 42: 776-82$.

69 ENGELHART MJ, GEERLINGS MI, RUITENBERG A, et al. Diet and risk of dementia : Does fat matter? The Rotterdam Study Neurology $2002 ; 59$ : 1915-21.

70 MORRIS MC, EVANS DA, BIENIAS JL, et al. Dietary fats and the risk of incident Alzheimer disease. Arch Neurol $2003 ; 60: 194-200$.

71 BARBERGER-GATEAU P, LETENNEUR L, DESCHAMPS V, PERES K, DARTIGUES JF, RENAUD S. Fish, meat, and risk of dementia : cohort study. BMJ $2002 ; 325: 932-3$.

72 MORRIS MC, EVANS DA, BIENIAS JL, et al. Consumption of fish and $\mathrm{n}-3$ fatty acids and risk of incident Alzheimer disease. Arch Neurol $2003 ; 60$ : 940-6.

73 OTSUKA M. Analysis of dietary factors in Alzheimer's disease : clinical use of nutritional intervention for prevention and treatment of dementia. Nippon Ronen Igakkai Zasshi $2000 ; 37$ : 9703. 
74 CONQUER JA, TIERNEY MC, ZECEVIC J, BETTGER WJ, FISHER RH. Fatty acid analysis of blood plasma of patients with Alzheimer's disease, other types of dementia, and cognitive impairment. Lipids $2000 ; 35: 1305-12$.

75 LAURIN D, VERREAULT R, LINDSAY J, DEWAILLY E, HOLUB BJ. Omega-3 fatty acids and risk of cognitive impairment and dementia. J Alzheimers Dis $2003 ; 5$ : 315-22.

76 OTSUKA M, YAMAGUCHI K, UEKI A. Similarities and differences between Alzheimer's disease and vascular dementia from the viewpoint of nutrition. Ann NY Acad Sci 2002 ; 977 : 155-1551.

77 KALMIJN S, FOLEY D, WHITE L, et al. Metabolic cardiovascular syndrome and risk of dementia in Japanese-American elderly men. The Honolulu-Asia aging study. Arterioscler Thromb Vasc Biol 2000 ; $20: 2255-60$.

78 SIMOPOULOS AP. Omega-3 fatty acids in inflammation and autoimmune diseases. J Am Coll Nutr $2002 ; 21: 495-505$.

79 YEHUDA S, RABINOVITZ S, CARASSO RL, MOSTOFSKY DI. Essential fatty acids preparation (SR-3) improves Alzheimer's patients quality of life. Int J Neurosci 1996 ; 87 : 141-9.

80 ZEROUGA M, BEAUGE F, NIEL E, DURAND G, BOURRE JM. Interactive effects of dietary (n-3) polyunsaturated fatty acids and chronic ethanol intoxication on synaptic membrane lipid composition and fluidity in rats. Biochim Biophys Acta $1991 ; 1086$ : 295-304.

81 HORROBIN DF. Schizophrenia : the illness that made us human. Med Hypotheses $1998 ; 50: 269-$ 88.

82 PEET M, LAUGHARNE JD, MELLOR J, RAMCHAND CN. Essential fatty acid deficiency in erythrocyte membranes from chronic schizophrenic patients, and the clinical effects of dietary supplementation. Prostaglandins Leukot Essent Fatty Acids 1996 ; 55 : 71-5.

83 ASSIES J, LIEVERSE R, VREKEN P, WANDERS RJ, DINGEMANS PM, LINSZEN DH. Significantly reduced docosahexaenoic and docosapentaenoic acid concentrations in erythrocyte membranes from schizophrenic patients compared with a carefully matched control group. Biol Psychiatry $2001 ; 49$ : 510-22.

84 HIBBELN JR, MAKINO KK, MARTIN CE, DICKERSON F, BORONOW J, FENTON WS. Smoking, gender, and dietary influences on erythrocyte essential fatty acid composition among patients with schizophrenia or schizoaffective disorder. Biol Psychiatry $2003 ; 53: 431-41$.

85 FENTON WS, HIBBELN J, KNABLE M. Essential fatty acids, lipid membrane abnormalities, and the diagnosis and treatment of schizophrenia. Biol Psychiatry $2000 ; 47: 8-21$.

86 LAUGHARNE JD, MELLOR JE, PEET M. Fatty acids and schizophrenia. Lipids 1996 ; 31 : S163-S165.

87 ARVINDAKSHAN M, GHATE M, RANJEKAR PK, EVANS DR, MAHADIK SP. Supplementation with a combination of omega-3 fatty acids and antioxidants (vitamins $E$ and $C$ ) improves the outcome of schizophrenia. Schizophr Res $2003 ; 62$ : 195-204. 
88 PURI BK, RICHARDSON AJ, HORROBIN DF, et al. Eicosapentaenoic acid treatment in schizophrenia associated with symptom remission, normalisation of blood fatty acids, reduced neuronal membrane phospholipid turnover and structural brain changes. Int J Clin Pract 2000 ; 54 : 57-63.

89 PEET M, BRIND J, RAMCHAND CN, SHAH S, VANKAR GK. Two double-blind placebo-controlled pilot studies of eicosapentaenoic acid in the treatment of schizophrenia. Schizophr Res $2001 ; 49: 243-51$.

90 EMSLEY R, MYBURGH C, OOSTHUIZEN P, VAN RENSBURG SJ. Randomized, placebo-controlled study of ethyl-eicosapentaenoic acid as supplemental treatment in schizophrenia. Am J Psychiatry $2002 ; 159: 1596-8$.

91 FENTON WS, DICKERSON F, BORONOW J, HIBBELN JR, KNABLE M. A placebo-controlled trial of omega-3 fatty acid (ethyl eicosapentaenoic acid) supplementation for residual symptoms and cognitive impairment in schizophrenia. Am J Psychiatry $2001 ; 158$ : 2071-4.

92 HORROBIN DF. Omega-3 Fatty acid for schizophrenia. Am J Psychiatry 2003 ; 160 : 188-9.

93 PEET M. Eicosapentaenoic acid in the treatment of schizophrenia and depression : rationale and preliminary double-blind clinical trial results. Prostaglandins Leukot Essent Fatty Acids $2003 ; 69$ : 477-85.

94 HALS J, BJERVE KS, NILSEN H, SVALASTOG AG, EK J. Essential fatty acids in the nutrition of severely neurologically disabled children. Br J Nutr $2000 ; 83: 219-25$.

95 VALLAT JM, BOURRE JM, DUMONT O, et al. Case of dementia and myoclonia in an adult associated with anomalies in polyunsaturated fatty acids in leukocytes and peripheral nerve. An ultrastructural study of peripheral nerve. Eur Neurol $1985 ; 24: 208-16$.

96 MARTINEZ M. Restoring the DHA levels in the brains of Zellweger patients. J Mol Neurosci 2001 ; $16: 309-16$.

97 RICHARDSON AJ, ROSS MA. Fatty acid metabolism in neurodevelopmental disorder : a new perspective on associations between attention-deficit/hyperactivity disorder, dyslexia, dyspraxia and the autistic spectrum. Prostaglandins Leukot Essent Fatty Acids $2000 ; 63: 1-9$.

98 RICHARDSON AJ, CALVIN CM, CLISBY C, et al. Fatty acid deficiency signs predict the severity of reading and related difficulties in dyslexic children. Prostaglandins Leukot Essent Fatty Acids 2000 ; $63: 69-74$.

99 TAYLOR KE, HIGGINS CJ, CALVIN CM, et al. Dyslexia in adults is associated with clinical signs of fatty acid deficiency. Prostaglandins Leukot Essent Fatty Acids $2000 ; 63$ : 75-8.

100 RICHARDSON AJ, PURI BK. The potential role of fatty acids in attention-deficit/hyperactivity disorder. Prostaglandins Leukot Essent Fatty Acids $2000 ; 63: 79-87$. 
101 ROSS BM, MCKENZIE I, GLEN I, BENNETT CP. Increased levels of ethane, a non-invasive marker of n-3 fatty acid oxidation, in breath of children with attention deficit hyperactivity disorder. Nutr Neuros $2003 ; 6: 277-81$.

102 STEVENS L, ZHANG W, PECK L, et al. EFA supplementation in children with inattention, hyperactivity, and other disruptive behaviors. Lipids $2003 ; 38: 1007-21$.

103 VOIGT RG, LLORENTE AM, JENSEN CL, FRALEY JK, BERRETTA MC, HEIRD WC. A randomized, double-blind, placebo-controlled trial of docosahexaenoic acid supplementation in children with attention-deficit/hyperactivity disorder. J Pediatr $2001 ; 139: 189-96$.

104 VANCASSEL S, DURAND G, BARTHELEMY C, et al. Plasma fatty acid levels in autistic children. Prostaglandins Leukot Essent Fatty Acids $2001 ; 65$ : 1-7.

105 BELL JG, SARGENT JR, TOCHER DR, DICK JR. Red blood cell fatty acid compositions in a patient with autistic spectrum disorder : a characteristic abnormality in neurodevelopmental disorders? Prostaglandins Leukot Essent Fatty Acids 2000 ; 63 : 21-5.

106 JOHNSON SM, HOLLANDER E. Evidence that eicosapentaenoic acid is effective in treating autism. J Clin Psychiatry $2003 ; 64$ : 848-9.

107 PURI BK, SINGH I. Normal phospholipid-related signal transduction in autism. Prog Neuropsychopharmacol Biol Psychiatry $2002 ; 26$ : 1405-7.

108 BUYDENS-BRANCHEY L, BRANCHEY M, MCMAKIN DL, HIBBELN JR. Polyunsaturated fatty acid status and relapse vulnerability in cocaine addicts. Psychiatry Res $2003 ; 120: 29-35$.

109 BUYDENS-BRANCHEY L, BRANCHEY M, MCMAKIN DL, HIBBELN JR. Polyunsaturated fatty acid status and aggression in cocaine addicts. Drug Alcohol Depend $2003 ; 71$ : 319-23.

110 FRANCES H, GRAULET A, DEBRAY M, COUDEREAU JP, GUERIS J, BOURRE JM. Morphine-induced sensitization of locomotor activity in mice : effect of social isolation on plasma corticosterone levels. Brain Res $2000 ; 860$ : 136-40.

111 BATES D. Dietary lipids and multiple sclerosis. Ups J Med Sci 1990(Suppl 48) : 173-87.

112 DI BIASE A, SALVATI S. Exogenous lipids in myelination and myelination. Kaohsiung J Med Sci $1997 ; 13: 19-29$.

113 NORDVIK I, MYHR KM, NYLAND H, BJERVE KS. Effect of dietary advice and n-3 supplementation in newly diagnosed MS patients. Acta Neurol Scand $2000 ; 102$ : 143-9.

114 CALDER PC. n-3 polyunsaturated fatty acids and cytokine production in health and disease. Ann Nutr Metab 1997 ; 41 : 203-34. 
115 BAZAN NG. The metabolism of omega-3 polyunsaturated fatty acids in the eye : the possible role of docosahexaenoic acid and docosanoids in retinal physiology and ocular pathology. Prog Clin Biol Res $1989 ; 312$ : 95-112.

116 MARCHESELLI VL, HONG S, LUKIW WJ, et al. Novel docosanoids inhibit brain ischemiareperfusion-mediated leukocyte infiltration and pro-inflammatory gene expression. J Biol Chem $2003 ; 278: 43807-17$.

117 TURNER N, ELSE PL, HULBERT AJ. Docosahexaenoic acid (DHA) content of membranes determines molecular activity of the sodium pump : implications for disease states and metabolism. Naturwissenschaften $2003 ; 90: 521-3$.

118 GERBI A, ZEROUGA M, DEBRAY M, DURAND G, CHANEZ C, BOURRE JM. Effect of dietary alphalinolenic acid on functional characteristic of $\mathrm{Na}+/ \mathrm{K}(+)-A T P a s e$ isoenzymes in whole brain membranes of weaned rats. Biochim Biophys Acta $1993 ; 1165$ : 291-8.

119 GERBI A, ZEROUGA M, DEBRAY M, DURAND G, CHANEZ C, BOURRE JM. Effect of fish oil diet on fatty acid composition of phospholipids of brain membranes and on kinetic properties of $\mathrm{Na}+, \mathrm{K}(+)$ ATPase isoenzymes of weaned and adult rats. J Neurochem $1994 ; 62: 1560-9$.

120 PERICHON R, BOURRE JM, KELLY JF, ROTH GS. The role of peroxisomes in aging. Cell Mol Life Sci $1998 ; 54: 641-52$.

121 HEUDE B, DUCIMETIERE P, BERR C. Cognitive decline and fatty acid composition of erythrocyte membranes -The EVA Study. Am J Clin Nutr $2003 ; 77: 803-8$.

122 KALMIJN S, FESKENS EJ, LAUNER L, KROMHOUT D. Polyunsaturated fatty acids, antioxidants, and cognitive function in very old men. Am J Epidemiol $1997 ; 145$ : 33-41.

123 MARTIN DS, SPENCER P, HORROBIN DF, LYNCH MA. Long-term potentiation in aged rats is restored when the age-related decrease in polyunsaturated fatty acid concentration is reversed. Prostaglandins Leukot Essent Fatty Acids $2002 ; 67$ : 121-30.

124 YEHUDA S, RABINOVITZ S, CARASSO RL, MOSTOFSKY DI. The role of polyunsaturated fatty acids in restoring the aging neuronal membrane. Neurobiol Aging $2002 ; 23: 843-53$.

125 KIDD PM. A review of nutrients and botanicals in the integrative management of cognitive dysfunction. Altern Med Rev $1999 ; 4$ : 144-61.

126 HUNG MC, SHIBASAKI K, YOSHIDA R, SATO M, IMAIZUMI K. Learning behaviour and cerebral protein kinase $C$, antioxidant status, lipid composition in senescence-accelerated mouse : influence of a phosphatidylcholine-vitamin B12 diet. Br J Nutr $2001 ; 86$ : 163-71.

127 CARRIE I, GUESNET P, BOURRE JM, FRANCES H. Diets containing long-chain n-3 polyunsaturated fatty acids affect behaviour differently during development than ageing in mice. Br J Nutr $2000 ; 83$ : 439-47.

128 AFSSA. Acides gras de la famille oméga 3 et système cardiovasculaire : intérêt nutritionnel et allégations. Afssa, 10 juillet. 2003. 
129 LEGRAND P, BOURRE JM, DESCOMPS B, DURAND G, RENAUD S. In : Lipides Apports nutritionnels conseillés. Tec et Doc Lavoisier, $2000: 62-82$.

130 SU HM, BERNARDO L, MA XH, CORSO TN, NATHANIELZ PW, BRENNA JT. Bioequivalence of dietary alpha-linolenic acid an docosahexaenoic acids as a source of docosahexanenoate in brain and associated organs of neonatal baboon. Pediatr Res $1999 ; 45: 87-93$.

131 LEGRAND P. Données récentes sur les désaturases chez l'animal et l'homme. Cah Nutr Diét $2003 ; 38: 376-83$.

132 DESCOMPS B. Les désaturases au cours du développement chez l'homme. Cah Nutr Diét 2003 ; 38 : 384-91.

133 BURDGE G, FINNEGAN Y, MINIHANE A, WILLIAMS C, WOOTON S. Effect of altered dietary n-3 fatty acid intake upon plasma lipid fatty acid composition, conversion of (13C) alpha-linolenic acid to longer chain fatty acid and partitioning towards beta-oxidation in older men. British J Nutr $2003 ; 90$ : 311-21. 